Check for updates

Cite this: Mater. Chem. Front., 2020, 4, 2018

Received 28th March 2020 Accepted 13th May 2020

DOI: $10.1039 / \mathrm{d} 0 \mathrm{qm} 00190 \mathrm{~b}$

rsc.li/frontiers-materials

\section{The design of an extended multiple resonance TADF emitter based on a polycyclic amine/ carbonyl system + t}

\author{
Dianming Sun, ${ }^{\text {ab }}$ Subeesh Madayanad Suresh, ${ }^{\text {b }}$ David Hall, ${ }^{\text {bc }}$ Ming Zhang, ${ }^{a}$ \\ Changfeng Si, ${ }^{b}$ David B. Cordes, (D) ${ }^{b}$ Alexandra M. Z. Slawin, (D) b Yoann Olivier, (DD*cd \\ Xiaohong Zhang*a and Eli Zysman-Colman (iD *b
}

\begin{abstract}
The development of multiple resonance thermally activated delayed fluorescence (MR-TADF) materials possessing narrow emission spectra has attracted significant attention as emitters for high colour purity organic light emitting diodes (OLEDs). In this work, a simple design strategy is introduced to construct an MR-TADF emitter, DDiKTa, through dimerization of the known MR-TADF emitter DiKTa. This design permits concentration quenching to be largely suppressed, which is a known weakness of previously reported MR-TADF emitters. OLEDs based on DDiKTa show an EQE $E_{\max }$ of $19 \%$ at a doping concentration of $9 \mathrm{wt} \%$. The electroluminescence spectrum is red-shifted into the green, producing a rare example of a green-emitting MR-TADF OLED.
\end{abstract}

\section{Introduction}

Thermally activated delayed fluorescence (TADF) emitters have attracted significant attention ${ }^{1-5}$ as replacement candidates for phosphorescent organometallic complexes in organic light emitting diodes (OLEDs). Such materials are capable of recruiting $100 \%$ of the generated excitons for light production without the requirement of the heavy metal center to mediate intersystem crossing events between the excited singlet and triplet states. The most common strategy to achieve efficient TADF emitters is based on a twisted donor-acceptor molecular design $^{6}$ that promotes a reduction in the exchange integral between the highest occupied molecular orbital (HOMO) and lowest unoccupied molecular orbital (LUMO) due to localization of the electron density on the donor and acceptor moieties, respectively. Thus, a small energy gap between the lowest triplet

\footnotetext{
${ }^{a}$ Institute of Functional Nano \& Soft Materials (FUNSOM) and Jiangsu Key Laboratory for Carbon-Based Functional Materials \& Devices, Soochow University, Suzhou, Jiangsu 215123, P. R. China. E-mail: xiaohong_zhang@suda.edu.cn

${ }^{b}$ Organic Semiconductor Centre, EaStCHEM School of Chemistry, University of St Andrews, St Andrews KY16 9ST, UK. E-mail: eli.zysman-colman@st-andrews.ac.uk ${ }^{c}$ Laboratory for Chemistry of Novel Materials, University of Mons, 7000, Mons, Belgium

${ }^{d}$ Unité de Chimie Physique Théorique et Structurale \& Laboratoire de Physique du Solide, Namur Institute of Structured Matter, Université de Namur, Rue de Bruxelles, 61, 5000 Namur, Belgium

$\dagger$ Electronic supplementary information (ESI) available. CCDC 1992507. For ESI and crystallographic data in CIF or other electronic format see DOI: 10.1039/d0qm00190b \# The research data supporting this publication can be accessed at https://doi.org/10.17630/1bec3f8b-84ae-4a1c-87da-9d36465f811b.
}

and singlet excited states $\left(\Delta E_{\mathrm{ST}}\right)$ is achieved that then permits the thermal up-conversion of triplet excitons to singlet excitons through reverse intersystem crossing (RISC).

However, with few exceptions, ${ }^{7-11}$ the emission from TADF compounds is broad (typically, 0.45-0.55 eV; 80-110 nm), due to the charge-transfer (CT) character of the emission singlet state. ${ }^{12}$ This leads to poor colour purity in the OLED. With the increasing commercial requirements for high-resolution OLED displays, new red, green and blue emitters need to be developed that show both potential for $100 \%$ internal quantum efficiency and narrow emission spectra, obviating the use of color filters and their associated energy loss. ${ }^{13}$

Recently, a new approach to TADF emitter design was reported by Hatakeyama et al. based on $\mathrm{p}$ - and n-doped nanographene compounds. The first example was the rigid boron-oxygen polycyclic aromatic framework, DOBNA (see Fig. S1 for structures of literature emitters, ESI $\dagger$ ). ${ }^{14}$ The presence of para-disposed boron and oxygen atoms in the compound possessing complementary resonance effects led to significantly separated electron densities between the HOMO and LUMO and a correspondingly small $\Delta E_{\mathrm{ST}}$ of $0.15 \mathrm{eV}$. The emission of DOBNA unfortunately, is in the UV region, which was deemed too high in energy for this compound to be used as an emitter for OLEDs. The same group then reported the nitrogen-containing blue-emitting DABNA-1, which served as the first multiple resonance TADF (MR-TADF) emitter used in an OLED. ${ }^{15}$ OLEDs employing DABNA-1 exhibited an emission at $\lambda_{\mathrm{EL}}$ of $459 \mathrm{~nm}$ with an FWHM of $28 \mathrm{~nm}$ and the corresponding CIE coordinates of $(0.13,0.09)$, and with a maximum external quantum efficiency, $\mathrm{EQE}_{\max }$, of $13.5 \%$. Further improvement in the 
design was evidenced in DABNA-2, a suitably substituted analogue of DABNA-1, which produced a blue OLED with $\lambda_{\mathrm{EL}}$ of $467 \mathrm{~nm}$, an FWHM of $28 \mathrm{~nm}$ and CIE coordinates of $(0.12,0.13)$, but with a much-improved $\mathrm{EQE}_{\max }$ of $20.2 \%$. Inspired by these early achievements, several derivatives have been reported. With the addition of $t$-butyl groups on the DABNA-1 core, $\boldsymbol{t}$-DABNA shows a shorter delayed lifetime and a consequently improved OLED efficiency roll-off. ${ }^{16}$ By introducing carbazole, TBN-TPA exhibited a significantly improved performance with an $\mathrm{EQE}_{\max }$ of $32.1 \%$ for a blue OLED. ${ }^{17}$ The extended polycyclic framework of $\boldsymbol{\nu}$-DABNA results in a state-of-the-art deep blue OLED exhibiting an $\mathrm{EQE}_{\max }$ of $34.4 \%{ }^{18}$ Boranes are not the only acceptor motif used in MRTADF emitters. Recently, ketone-containing MR-TADF emitters have been shown to produce blue to sky-blue OLEDs. ${ }^{19,20}$ We showed that by decorating a MR-TADF emitter, DiKTa, with mesityl groups, Mes ${ }_{3}$ DiKTa, undesired aggregation caused quenching and excimer emission can be largely mitigated. ${ }^{21}$

Herein, we report the emitter DDiKTa, which is a dimeric compound consisting of two MR-TADF DiKTa units (Scheme 1). The slightly increased conjugation present in DDikTa compared to DiKTa contributes to a modest red-shifting of the emission; however, the new compound conserves its MR-TADF status. OLEDs employing DDiKTa showed blue-green emission with $\lambda_{\text {EL }}$ of $500 \mathrm{~nm}$ and CIE coordinates of $(0.18,0.53)$ and a high $\mathrm{EQE}_{\max }$ of $19 \%$ at a doping concentration of $9 \mathrm{wt} \%$ in DPEPO; at a doping concentration of $12 \mathrm{wt} \%$, the $\mathrm{EQE}_{\max }$ was $18.5 \%$. To the best of our knowledge, there exists only a single report of green MR-TADF OLEDs with $\lambda_{\mathrm{EL}}$ ranging from 493-501 $\mathrm{nm}$ and $\mathrm{EQE}_{\max }$ ranging from $21-22 \% .^{22}$ The results demonstrate how to design MR-TADF emitters exhibiting both a red-shifting of the emission and a suppression of concentration quenching in the solid state.

\section{Results and discussion}

\subsection{Synthesis}

DDiKTa was synthesized in five steps from dimethyl 2,2'(phenylazanediyl)dibenzoate (Scheme 1). Iodination with $\mathrm{N}$ iodosuccinimide (NIS) gave compound 1 in high yield (85\%). Saponification followed by acyl chloride formation with $\mathrm{SOCl}_{2}$, and Lewis-acid catalysed Friedel-Crafts acylation afforded key intermediate 2 in $42 \%$ yield, the structure of which was confirmed by single crystal X-ray diffraction (Fig. S16, ESI $\dagger$ ). Compound 2 was converted to the boronate ester 3 via palladium-catalyzed borylation, which was then coupled with

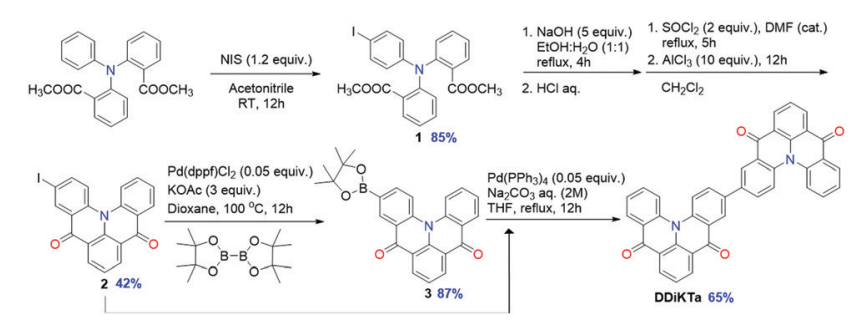

Scheme 1 Synthetic route for DDiKTa. a second equivalent of 2 under Suzuki-Miyaura cross-coupling conditions to afford DDiKTa in a moderate yield (65\%).

\subsection{Computational studies}

The frontier molecular orbitals (the HOMO, HOMO-1, LUMO and LUMO+1, see Fig. 1) isocontour plots of DDiKTa were obtained based on the optimized ground state gas-phase geometry using Density Functional Theory (DFT) with the PBE0 functional and the 6-31G(d,p) basis set. The calculated dihedral angle between the two DiKTa moieties in DDiKTa in the ground state is around $37^{\circ}$. The calculated HOMO and LUMO levels of DDiKTa are -6.04 and $-2.34 \mathrm{eV}$, respectively. Compared with the reference compound DiKTa, the HOMO level of DDiKTa is destabilized by $0.16 \mathrm{eV}$ while the LUMO is likewise stabilized by $0.11 \mathrm{eV}$, both a result of the increased conjugation in DDiKTa. The result is a smaller $\Delta E_{\mathrm{H}-\mathrm{L}}$ and an expected red-shift CT absorption compared to DiKTa as shown in Fig. S2 (ESI $\dagger$ ) according to simulated vertical excitation.

We recently showed the importance of using a suitably high level of theory in order to accurately predict the excited state energies of MR-TADF compounds. ${ }^{12}$ Calculations using the Spin-Component Scaling Coupled-Cluster second-order approximate Coupled-Cluster (SCS-CC2) method and the cc-pVDZ level reveal that the short-range charge-transfer character of DiKTa (Fig. S2, ESI $\dagger$ ) is inherited in DDiKTa. The calculated lowest singlet and triplet energy levels of DDiKTa are 3.39 and $3.12 \mathrm{eV}$, respectively, which are slightly lower than those of DiKTa $\left(\mathrm{S}_{1}=3.45 \mathrm{eV}, \mathrm{T}_{1}=3.18 \mathrm{eV}\right)$ and so DDiKTa is expected to show a red-shifted emission. As a result, the same $\Delta E_{\mathrm{ST}}$ of $0.27 \mathrm{eV}$ indicates similar triplet harvesting ability through RISC (see Tables S1 and S2 for a summary of the excited states calculations, ESI $\dagger$ ).

\subsection{Photophysical properties}

The electrochemical behavior of DDiKTa was studied by cyclic voltammetry (CV) and differential pulse voltammetry (DPV) in degassed dichloromethane (DCM) with $\left[n \mathrm{Bu}_{4} \mathrm{~N}\right] \mathrm{PF}_{6}$ as the supporting electrolyte, and the values reported versus SCE. The CV and DPV are shown in Fig. 2a and the data for oxidation/ reduction potentials $\left(E_{\mathrm{ox}} / E_{\mathrm{red}}\right)$ are summarized in Table S3 (ESI $\dagger$ ). DDiKTa shows irreversible anodic and reversible cathodic waves. The $E_{\text {ox }}$ and $E_{\text {red }}$ determined from the DPV peak

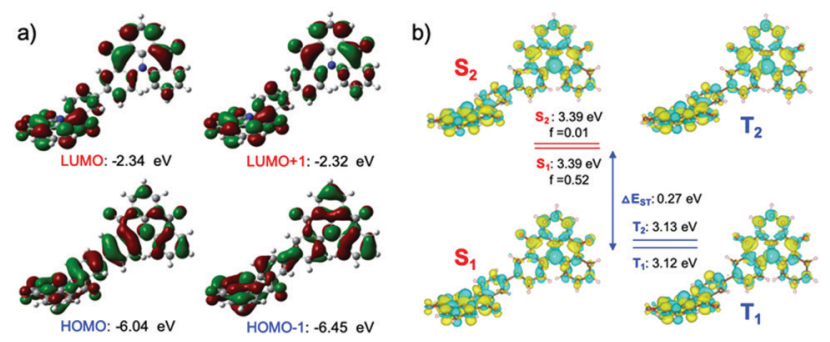

Fig. 1 (a) Isocontour plots (cutoff $=0.02$ a.u.) of the $\mathrm{HOMO} / \mathrm{HOMO}-1$ and LUMO/LUMO+1 orbitals calculated in the gas phase at the PBEO/6$31 \mathrm{G}(\mathrm{d}, \mathrm{p})$ level; (b) difference density plots of $\mathrm{S}_{1}, \mathrm{~S}_{2}, T_{1}$ and $\mathrm{T}_{2}$ excited states calculated in the gas phase at the SCS-CC2/cc-pVDZ level. $f$ indicates the oscillator strength. 



Fig. 2 (a) Cyclic voltammogram (CV) and differential pulse voltammetry (DPV) in degassed DCM with $0.1 \mathrm{M}\left[n \mathrm{Bu}_{4}{\mathrm{~N}] P F_{6}}_{6}\right.$ as the supporting electrolyte and $\mathrm{Fc} / \mathrm{Fc}^{+}$as the internal reference $(0.38 \mathrm{~V}$ vs. SCE). (b) Absorption and steady-state PL spectra obtained in toluene at RT and $77 \mathrm{~K}$, phosphorescence spectra obtained in toluene glass at $77 \mathrm{~K}$ (time delay of $70 \mathrm{~ms}$; time window of $140 \mathrm{~ms}$ ). (c) Comparison of steady-state PL in both aerated and degassed toluene. (d) Solvatochromatic PL study $\left(\lambda_{\text {exc }}=330 \mathrm{~nm}\right.$ ).

values are 1.63 and $-1.27 \mathrm{~V}$, respectively, showing the expected decreased $\Delta E$ due to increased conjugation compared to those of DiKTa $\left(E_{\text {ox }}: 1.65 \mathrm{eV} ; E_{\text {red }}:-1.33 \mathrm{eV} v s\right.$. SCE; determined from the DPV in MeCN), ${ }^{21}$ a trend that is consistent with the DFT calculations. The UV-vis absorption, fluorescence and phosphorescence spectra of DDiKTa in dilute toluene solution are depicted in Fig. 2b and the optoelectronic properties are summarized in Table S4 (ESI $\dagger$ ). The absorption spectrum exhibits two major absorption bands. The high-energy band from $300-380 \mathrm{~nm}$ originates from a $\pi-\pi^{*}$ transition of the aromatic skeleton while the broad, low-energy band at $440 \mathrm{~nm}$ arises from short-range charge transfer (SRCT) transitions that are characteristic of MR-TADF molecules. Compared to the SRCT absorption band of DiKTa at $\lambda_{\mathrm{abs}}$ of $435 \mathrm{~nm},{ }^{20}$ that of DDiKTa is (i) red-shifted and (ii) broader. The red-shifted emission is supported by SCS-CC2 calculations and the broadened PL spectrum is likely explained by the likely large conformational landscape accessible at room temperature for the torsion between the two DiKTa moieties. The room temperature steady-state emission spectrum of DDiKTa is similarly red-shifted with $\lambda_{\mathrm{PL}}$ at $470 \mathrm{~nm}$, less structured than DiKTa and adversely broadened (FWHM from $27 \mathrm{~nm} / 1251 \mathrm{~cm}^{-1}$ for DiKTa to $47 \mathrm{~nm} / 1639 \mathrm{~cm}^{-1}$ for DDiKTa). The mirror image emission spectrum of the absorption spectrum and the small Stokes shift of $26 \mathrm{~nm} / 1291 \mathrm{~cm}^{-1}$ suggests a small geometry relaxation in the singlet state (Fig. 2b), highlighting the quite rigid structure of DDiKTa. The rigid nature of the compound is also responsible for the narrow emission spectrum observed at RT.

A similar profile but with more pronounced vibronic progression is observed for the phosphorescence spectrum obtained after $70 \mathrm{~ms}$ at $77 \mathrm{~K}$ in a toluene glass. The $\Delta E_{\mathrm{ST}}$, determined from the peak maxima of the fluorescence and
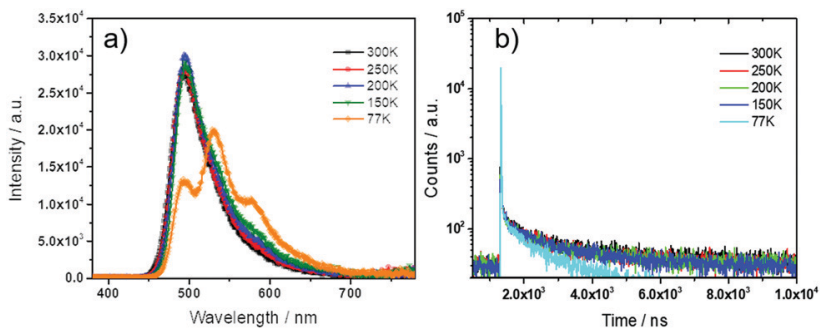

Fig. 3 Temperature-dependent (a) steady-state PL spectra and (b) transient decay characteristics of $9 \mathrm{wt} \%$ DDiKTa doped into a DPEPO matrix $\left(\lambda_{\mathrm{exc}}=330 \mathrm{~nm}\right)$.

phosphorescence spectra, of $0.21 \mathrm{eV}$ is sufficiently small to enable a RISC process at room temperature. Fig. 2c shows the oxygen dependency on the intensity of the steady-state PL spectra. The significant drop off in intensity in the emission measured in aerated toluene compared to that after degassing with $\mathrm{N}_{2}$ clearly demonstrates the contribution from triplet excitons. Similar to DiKTa and Mes3DiKTa, a modest positive solvatochromism was observed in Fig. $2 \mathrm{~d}$ in the steady state PL spectra, reflective of the short-range CT state present in MRTADF emitters.

DPEPO was identified as a suitable host matrix for OLEDs due to its high triplet energy and appropriate HOMO/LUMO levels. The steady-state and transient PL spectra were therefore investigated in $9 \mathrm{wt} \%$ doped films of DDiKTa in a DPEPO matrix. This concentration was chosen as it corresponds to the doping concentration used in the optimized OLED. As shown in Fig. S5 (ESI $\dagger$ ), a similar oxygen dependence on the intensity is also observed, analogous to that seen in toluene. The temperature-dependent steady-state emission of DDiKTa in DPEPO is shown in Fig. 3a. The similar intensity of the PL indicates an unexpected temperature independence within the temperature range of $300-150 \mathrm{~K}$, while at $77 \mathrm{~K}$ there is a redshifted and structured emission that is reminiscent of the steady-state emission obtained in toluene glass at $77 \mathrm{~K}$


Fig. 4 (a) Device configuration and energy levels for each layer; (b) $J-V-L$ characteristics; (c) EQE and PE versus luminance characteristics; (d) electroluminescent spectra for devices with various doping concentrations of DDiKTa (inset figure shows the CIE coordinates of four devices). 
Table 1 Summary of device performance ${ }^{a}$



${ }^{a}$ The device structure is: ITO/TAPC $(35 \mathrm{~nm}) / \mathrm{TCTA}(10 \mathrm{~nm}) / \mathrm{CzSi}(10 \mathrm{~nm}) / \mathrm{DPEPO}: x$ wt\% DDiKTa(20 nm)/TmPyPB$(40 \mathrm{~nm}) / \mathrm{LiF}(1 \mathrm{~nm}) / \mathrm{Al} .{ }^{b} \mathrm{At} \mathrm{a}$ luminance of $1 \mathrm{~cd} \mathrm{~m}^{-2}$.

(Fig. 2b). The steady-state emission at $77 \mathrm{~K}$ in DPEPO is a mixture of fluorescence and phosphorescence, which was confirmed by comparing the $77 \mathrm{~K}$ prompt fluorescence spectrum integrated by iCDD (time delay after the laser excitation of $0 \mathrm{~ns}$; gate width of $100 \mathrm{~ns}$ ) and the phosphorescence spectrum integrated by iCDD with a time delay of $1 \mathrm{~ms}$ laser excitation and a gate width of $100 \mathrm{~ms}$ (Fig. S4b, ESI $\dagger$ ). The $\Delta E_{\mathrm{ST}}$ of $0.16 \mathrm{eV}$ in DPEPO, estimated from the onset of the prompt and phosphorescence spectra, is smaller than that measured in toluene glass $(0.21 \mathrm{eV})$, implying a more efficient RISC process in the former host medium; the activation energy $\Delta E_{\mathrm{a}}^{\mathrm{TADF}}$ is estimated to be $40 \mathrm{meV}$ (Fig. S6, ESI $\dagger$ ). The small activation energy in DPEPO may indicate the involvement of higher-lying excited states in the RISC process. Fig. S4a (ESI $\dagger$ ) shows that at room temperature, the prompt emission (time delay of $0 \mathrm{~ns}$; gate width of $10 \mathrm{~ns}$ ) and the delayed emission (time delay of $1 \mu \mathrm{s}$; gate width of $10 \mu \mathrm{s}$ ) align, indicating emission from the $\mathrm{S}_{1}$ state. The temperature-dependent transient PL (Fig. 3b) reveals a slight decrease of the delayed component with decreasing temperature, indicative of typical TADF behavior.

\subsection{Organic light-emitting diodes}

To evaluate the potential of DDiKTa as an emitter in OLEDs we fabricated several devices, modulating the doping concentration from $3 \mathrm{wt} \%$ to $12 \mathrm{wt} \%$, with DPEPO acting as the host matrix. The devices were fabricated with a configuration of: ITO/TAPC $(35 \mathrm{~nm}) /$ TCTA $(10 \mathrm{~nm}) / \mathrm{CzSi}(10 \mathrm{~nm}) / x$ wt\% DDiKTa: DPEPO $(20 \mathrm{~nm}) / \mathrm{TmPyPB}(40 \mathrm{~nm}) / \mathrm{LiF}(1 \mathrm{~nm}) / \mathrm{Al}(100 \mathrm{~nm})$ (Fig. 4a), where indium tin oxide (ITO) is the anode, and 4,4'cyclohexylidenebis[N,N-bis(4-methylphenyl)benzenamine] (TAPC) and tris(4-carbazoyl-9-ylphenyl)amine (TCTA) act as hole injection and transport layers, respectively. 9-(4-tert-Butylphenyl)3,6-bis(triphenylsilyl)-9H-carbazole (CzSi) is used to block excitons and DPEPO is used as the host, 1,3,5-tri $(m$-pyridin-3ylphenyl)benzene (TmPyPB) acts as the electron-transporting material, and LiF modifies the work function of the aluminum cathode.

Fig. 4 b shows the current density-voltage-luminance $(J-V-L)$ characteristics for the four devices. Device 1 with $3 \mathrm{wt} \%$ of DDiKTa shows a relatively high turn-on voltage at $5.7 \mathrm{~V}$ $\left(1 \mathrm{~cd} \mathrm{~m}^{-2}\right)$, which reveals poor charge injection and mobility in the emitting layer. However, with increasing doping concentration, the turn-on voltage gradually drops to $3.3 \mathrm{~V}$, which can be attributed to the better charge transporting ability of DDiKTa than that of DPEPO. Because of the imbalanced charge injection in Device 1, only an $\mathrm{EQE}_{\max }$ of $9.5 \%$ is obtained. Efficiencies can be optimized by increasing the doping concentration over $7 \mathrm{wt} \%$. The best performance obtained at $9 \mathrm{wt} \%$, Device 3, indicates a significant suppression of concentration quenching not normally observed in MR-TADF OLEDs. The $\mathrm{EQE}_{\max }$ for Devices 3 is $19 \%$ with CIE coordinates of $(0.18,0.53)$, Table 1 . Increasing the doping concentration to $12 \mathrm{wt} \%$ does not lead to further improvement in performance where the $\mathrm{EQE}_{\max }$ of Device 4 is $18.5 \%$.

The electroluminescence (EL) spectra for these devices exhibit modest FWHM from 55 to $59 \mathrm{~nm}$ that increase with increasing doping concentration. The CIE chromaticity coordinates shift from $(0.16,0.47)$ for Device 1 to $(0.19,0.54)$ for Device 4 ; all OLEDs show green emission (Fig. 4d). In comparison with the EL of DiKTa, the red-shifted EL of DDiKTa provides a simple design for accessing narrow green MR-TADF OLEDs.

\section{Conclusions}

In summary, an MR-TADF emitter DDiKTa has been developed through a simple dimerization of the previously reported emitter DiKTa. This design maintained the multi-resonance TADF photophysical features of its corresponding monomer DiKTa. Importantly, the weakly electronically coupled DiKTa fragments that result from the twisted confirmation in DDiKTa lead to both a red-shifting of the emission and a suppression of concentration quenching, a commonly encountered problem in rigid flat molecules that are emblematic of MR-TADF emitters. The best performing OLED exhibited an $\mathrm{EQE}_{\max }$ of $19 \%$ with an EL maximum of $500 \mathrm{~nm}$. This emitter design indicates the potential to maintain the desirable MR-TADF character of the emitter while extending the conjugation, providing an avenue to MR-TADF emitters in the green and beyond.

\section{Conflicts of interest}

There are no conflicts to declare.

\section{Acknowledgements}

This project has received funding from the European Union's Horizon 2020 research and innovation programme under the Marie Skłodowska Curie grant agreement No. 838009 (TSFP) and No. 838885 (NarrowbandSSL). D. S. acknowledges support from the Marie Skłodowska-Curie Individual Fellowship, the National Postdoctoral Program for Innovative Talents (BX201700164), and the Jiangsu Planned Projects for Postdoctoral Research Funds (2018K011A). The St Andrews team would also like to thank the 
Leverhulme Trust (RPG-2016047) and EPSRC (EP/P010482/1) for financial support. We thank Umicore AG for the gift of materials. Computational resources have been provided by the Consortium des Équipements de Calcul In-tensif (CÉCI), funded by the Fonds de la Recherche Scientifiques de Belgique (F. R. S.-FNRS) under Grant no. 2.5020.11. YO acknowledge fruitful discussion with Prof. D. Beljonne from the University of Mons, Prof. Juan-Carlos Sancho-Garcia from the University of Alicante and Prof. L. Muccioli from the University of Bologna.

\section{Notes and references}

1 Y. Tao, K. Yuan, T. Chen, P. Xu, H. Li, R. Chen, C. Zheng, L. Zhang and W. Huang, Thermally Activated Delayed Fluorescence Materials Towards the Breakthrough of Organoelectronics, Adv. Mater., 2014, 26(47), 7931-7958.

2 M. Y. Wong and E. Zysman-Colman, Purely Organic Thermally Activated Delayed Fluorescence Materials for Organic Light-Emitting Diodes, Adv. Mater., 2017, 29(22), 1605444.

3 Y. Liu, C. Li, Z. Ren, S. Yan and M. R. Bryce, All-organic thermally activated delayed fluorescence materials for organic light-emitting diodes, Nat. Rev. Mater., 2018, 3(4), 18020.

4 Y. Olivier, J. C. Sancho-Garcia, L. Muccioli, G. D'Avino and D. Beljonne, Computational Design of Thermally Activated Delayed Fluorescence Materials: The Challenges Ahead, J. Phys. Chem. Lett., 2018, 9(20), 6149-6163.

5 X. Liang, Z.-L. Tu and Y.-X. Zheng, Thermally Activated Delayed Fluorescence Materials: Towards Realization of High Efficiency Through Strategic Small Molecular Design, Chem. - Eur. J., 2019, 25(22), 5623-5642.

6 Y. Im, M. Kim, Y. J. Cho, J.-A. Seo, K. S. Yook and J. Y. Lee, Molecular Design Strategy of Organic Thermally Activated Delayed Fluorescence Emitters, Chem. Mater., 2017, 29(5), 1946-1963.

7 Y. J. Cho, S. K. Jeon, S.-S. Lee, E. Yu and J. Y. Lee, Donor Interlocked Molecular Design for Fluorescence-like Narrow Emission in Deep Blue Thermally Activated Delayed Fluorescent Emitters, Chem. Mater., 2016, 28(15), 5400-5405.

8 I. S. Park, K. Matsuo, N. Aizawa and T. Yasuda, HighPerformance Dibenzoheteraborin-Based Thermally Activated Delayed Fluorescence Emitters: Molecular Architectonics for Concurrently Achieving Narrowband Emission and Efficient Triplet-Singlet Spin Conversion, Adv. Funct. Mater., 2018, 28(34), 1802031.

9 Y. Im, S. H. Han and J. Y. Lee, Deep blue thermally activated delayed fluorescent emitters using $\mathrm{CN}$-modified indolocarbazole as an acceptor and carbazole-derived donors, J. Mater. Chem. C, 2018, 6(18), 5012-5017.

10 D. H. Ahn, S. W. Kim, H. Lee, I. J. Ko, D. Karthik, J. Y. Lee and J. H. Kwon, Highly efficient blue thermally activated delayed fluorescence emitters based on symmetrical and rigid oxygen-bridged boron acceptors, Nat. Photonics, 2019, 13(8), 540-546.

11 P. Rajamalli, N. Senthilkumar, P. Y. Huang, C. C. Ren-Wu, H. W. Lin and C. H. Cheng, New Molecular Design Concurrently
Providing Superior Pure Blue, Thermally Activated Delayed Fluorescence and Optical Out-Coupling Efficiencies, J. Am. Chem. Soc., 2017, 139(32), 10948-10951.

12 A. Pershin, D. Hall, V. Lemaur, J.-C. Sancho-Garcia, L. Muccioli, E. Zysman-Colman, D. Beljonne and Y. Olivier, Highly emissive excitons with reduced exchange energy in thermally activated delayed fluorescent molecules, Nat. Commun., 2019, 10(1), 597.

13 J. P. Spindler, T. K. Hatwar, M. E. Miller, A. D. Arnold, M. J. Murdoch, P. J. Kane, J. E. Ludwicki, P. J. Alessi and S. A. Van Slyke, System considerations for RGBW OLED displays, J. Soc. Inf. Disp., 2006, 14(1), 37-48.

14 H. Hirai, K. Nakajima, S. Nakatsuka, K. Shiren, J. Ni, S. Nomura, T. Ikuta and T. Hatakeyama, One-Step Borylation of 1, 3-Diaryloxybenzenes Towards Efficient Materials for Organic Light-Emitting Diodes, Angew. Chem., Int. Ed., 2015, 54(46), 13581-13585.

15 T. Hatakeyama, K. Shiren, K. Nakajima, S. Nomura, S. Nakatsuka, K. Kinoshita, J. Ni, Y. Ono and T. Ikuta, Ultrapure blue thermally activated delayed fluorescence molecules: efficient HOMO-LUMO separation by the multiple resonance effect, Adv. Mater., 2016, 28(14), 2777-2781.

16 S. H. Han, J. H. Jeong, J. W. Yoo and J. Y. Lee, Ideal blue thermally activated delayed fluorescence emission assisted by a thermally activated delayed fluorescence assistant dopant through a fast reverse intersystem crossing mediated cascade energy transfer process, J. Mater. Chem. C, 2019, 7(10), 3082-3089.

17 X. Liang, Z.-P. Yan, H.-B. Han, Z.-G. Wu, Y.-X. Zheng, H. Meng, J.-L. Zuo and W. Huang, Peripheral amplification of multi-resonance induced thermally activated delayed fluorescence for highly efficient OLEDs, Angew. Chem., Int. Ed., 2018, 130(35), 11486-11490.

18 Y. Kondo, K. Yoshiura, S. Kitera, H. Nishi, S. Oda, H. Gotoh, Y. Sasada, M. Yanai and T. Hatakeyama, Narrowband deepblue organic light-emitting diode featuring an organoboronbased emitter, Nat. Photonics, 2019, 13(10), 678-682.

19 X. Li, Y. Shi, K. Wang, M. Zhang, C.-J. Zheng, D.-M. Sun, G. Dai, X.-C. Fan, D.-Q. Wang and W. Liu, Thermally Activated Delayed Fluorescence Carbonyl Derivatives for Organic Light Emitting Diodes with Extremely Narrow Full-Width at Half-Maximum, ACS Appl. Mater. Interfaces, 2019, 11(14), 13472-13480.

20 Y. Yuan, X. Tang, X. Y. Du, Y. Hu, Y. J. Yu, Z. Q. Jiang, L. S. Liao and S. T. Lee, The Design of Fused Amine/ Carbonyl System for Efficient Thermally Activated Delayed Fluorescence: Novel Multiple Resonance Core and Electron Acceptor, Adv. Opt. Mater., 2019, 7(7), 1801536.

21 D. Hall, S. M. Suresh, P. L. dos Santos, E. Duda, S. Bagnich, A. Pershin, P. Rajamalli, D. B. Cordes, A. M. Slawin and D. Beljonne, Improving Processability and Efficiency of Resonant TADF Emitters: A Design Strategy, Adv. Opt. Mater., 2020, 8(2), 1901627.

22 Y. Zhang, D. Zhang, J. Wei, Z. Liu, Y. Lu and L. Duan, MultiResonance Induced Thermally Activated Delayed Fluorophores for Narrowband Green OLEDs, Angew. Chem., Int. Ed., 2019, 131(47), 17068-17073. 